
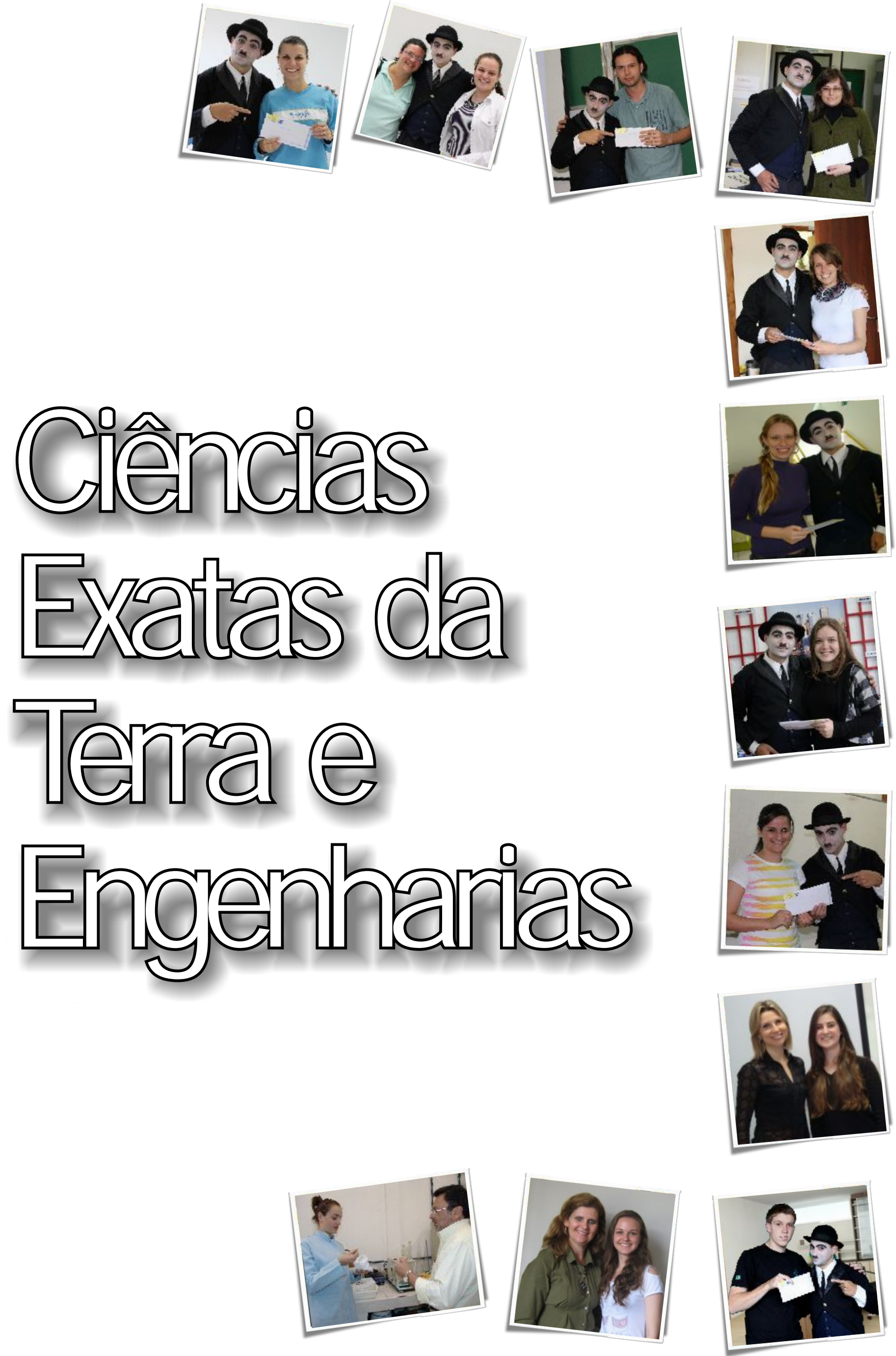


\section{ÁREA DE CIÊNCIAS EXATAS, DA TERRA E ENGENHARIAS}

Na área de Ciências Exatas, da Terra e Engenharias entre os 65 trabalhos apresentados no evento, $97 \%$ foram de alunos bolsistas de Iniciação Científica da Universidade, sendo 02 trabalhos de alunos de Iniciação Científica vinculados a outras Instituições de Ensino Superior do Estado do Rio Grande do Sul. A maioria dos trabalhos nesta área foi de bolsistas do Programa UNISC de Iniciação Científica - PUIC, seguida dos Programas de bolsa de verba externa para pagamentos de bolsas em projetos de pesquisa e Programa PIBIC/CNPq e PROBIC/FAPERGS. Nesta área também se observa a participação de estudantes participantes do Programa PUIC voluntário, dados demonstrados na Figura 03.

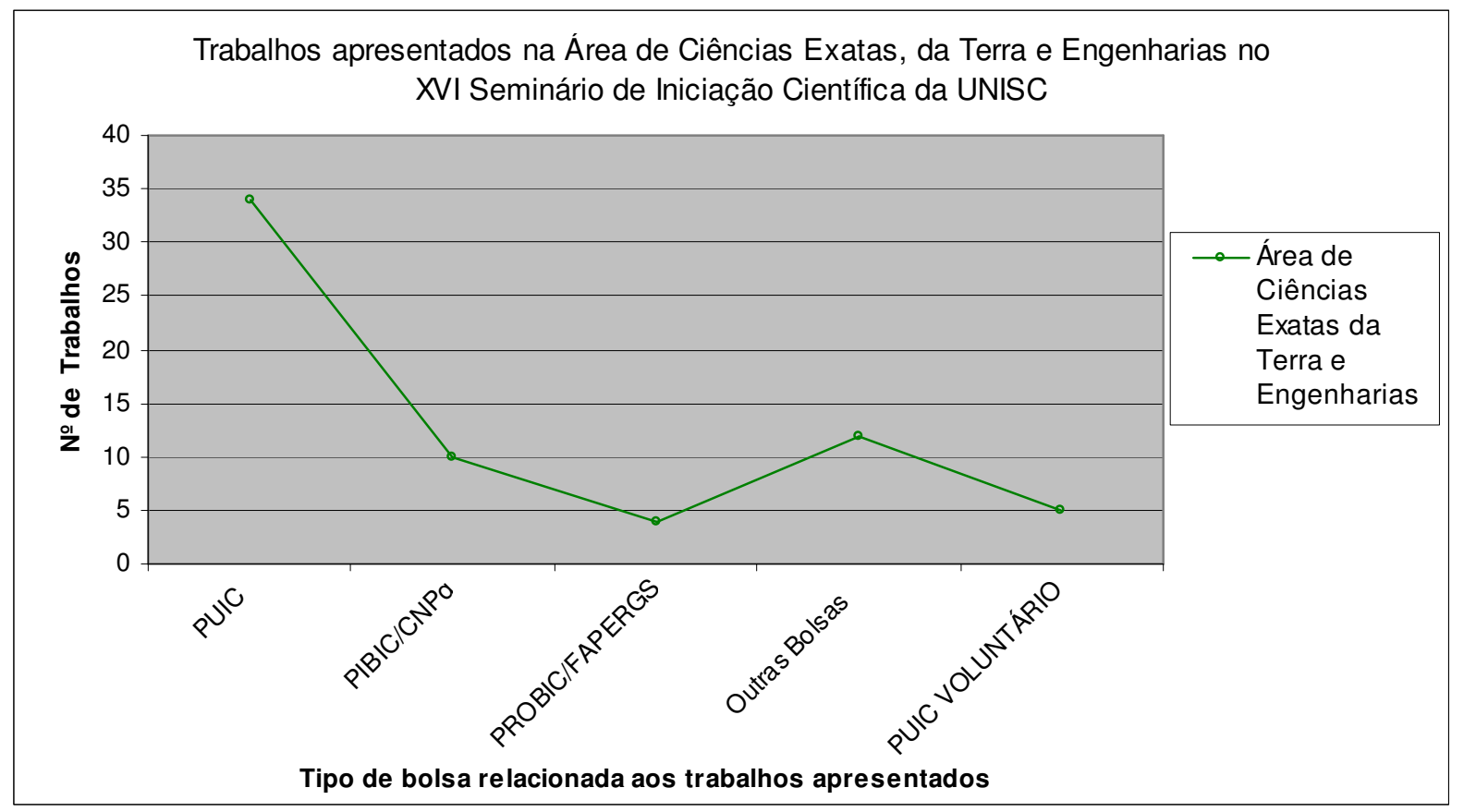

Figura 03 - Modalidade de bolsas dos estudantes participantes do XVI Seminário de Iniciação Científica na Área de Ciências Exatas da Terra e Engenharias.

Fonte: Coordenação de Pesquisa, UNISC, 2010. 


\title{
AVALIAÇAO DA QUALIDADE DAS ÁGUAS SUBTERRÂNEAS DA BACIA HIDROGRÁFICA DO RIO PARDO, SISTEMA AQUÍFERO GUARANI, RS, BRASIL
}

\author{
Marluce Purper ${ }^{1}$ \\ Marco Antonio Fontoura Hansen ${ }^{2}$ \\ Adilson Ben da Costa ${ }^{1}$ \\ Roberta Cristina Kaufmann ${ }^{1}$ \\ Ana Paula Wetzel ${ }^{1}$ \\ Alcido Kirst ${ }^{1}$ \\ Eduardo A. Lobo'
}

\section{RESUMO}

O objetivo desta pesquisa foi avaliar a qualidade das águas subterrâneas em áreas de preservação permanente (Sistema Aquífero Guarani - SAG) da Bacia Hidrográfica do Rio Pardo, RS, Brasil, através de variáveis físicas, químicas e microbiológicas. Nove pontos de coleta foram distribuídos ao longo da bacia, nos quais as águas subterrâneas foram classificadas quanto aos íns de maior ocorrência quantitativa. Os resultados indicaram que a maioria dos poços avaliados enquadraram-se na Classe 4 de usos da água, correspondendo a águas de usos menos restritivos (como recreação de contato secundário). Entretanto, deve-se considerar que os aquíferos são caracterizados por diferentes condições geológicas, com características físicas, químicas e biológicas intrínsecas, e também variações hidrogeoquímicas, sendo necessário que as suas classes de qualidade sejam determinadas com base nessas especificidades. As amostras P1, P2, P3, P5 e P9 classificaram-se como bicarbonatadas cálcicas; as águas dos pontos P4, P6, P7 como bicarbonatadas sódicas; e P8 como sulfatada. Verificouse que a qualidade das águas de poços com profundidade inferior a $6 \mathrm{~m}$ está mais vulnerável, devido a alterações antrópicas em função da concentração de nitrato, coliformes totais e termotolerantes, enquanto a qualidade das águas de poços mais profundos depende basicamente de suas características hidrogeológicas e hidrogeoquímicas naturais, em função das variáveis sulfato e sódio.

1 Laboratório de Limnologia. Universidade de Santa Cruz do Sul, UNISC, RS Av. Independência, 2293 - Bairro Universitário - Santa Cruz do Sul - Rio Grande do Sul - Código Postal: 96815-900 - Brasil. Tel.: +55 (51) 3711-3465 - Fax: +55 (51) 3717-7382. E-mail: marlucepurper@yahoo.com.br

2 Universidade Federal do Pampa - UNIPAMPA, RS. 
Palavras-chave: Bacia Hidrográfica do Rio Pardo. RS. Qualidade da água subterrânea. Sistema Aquífero Guarani.

\section{ABSTRACT}

The aim of this research was to evaluate the quality of groundwater in areas of permanent preservation (Guarani Aquifer System - GAS) in the Rio Pardo Hydrographical Basin, RS, Brazil, using physical, chemical and microbiological variables. Nine sampling points were distributed throughout the basin, where groundwater was classified according to their quantitative occurrence of ions. The results indicated that most of the wells evaluated were classified in Class 4 of water uses, corresponding to waters with less restrictive uses (such as secondary contact recreation). However, it should be considered that the aquifers are characterized by different geological conditions, with physical, chemical and biological intrinsical characteristics, and also hydrogeochemical variations, requiring that their quality levels are determined based on these specifications. Samples P1, P2, P3, P5 and P9 were classified as calcium bicarbonate, the waters of the points P4, P6, P7 as sodium bicarbonate and P8 as sulfated. It was found that the quality of water from wells with depths less than $6 \mathrm{~m}$ are more vulnerable due to anthropogenic activities, as showing by the concentration of nitrate, total and thermotolerant coliforms, while the quality of water from deeper wells basically depends on their hydrogeological and hydrogeochemical characteristics based on the concentration of sodium and sulfate variables.

Keywords: Groundwater quality. Guarani Aquifer System. Rio Pardo Hydrographical Basin. RS.

\section{INTRODUÇÃO}

O Sistema Aquífero Guarani (SAG) é um dos maiores reservatórios de água subterrânea do mundo, com estimativa de $50 \mathrm{mil}$ km3 de água doce armazenada em uma área total de 1,2 milhão de $\mathrm{km} 2$ distribuída em oito estados brasileiros e outros três países (Uruguai, Paraguai e Argentina) (BORGHETTI et al., 2004). Além de conter a maior parte das reservas subterrâneas, o Brasil também conta com muitas áreas de recarga, responsáveis pelo reabastecimento deste aquífero amplamente compartimentado por sistemas de falhas, o que aumenta ainda mais a responsabilidade brasileira em evitar a contaminação deste aquífero. Apesar disso, e da recomendação de que as zonas de recarga de um aquífero sejam consideradas áreas de proteção permanente, tem-se constatado que as culturas de cana-de-açúcar, soja e milho representam as principais atividades responsáveis pelos casos de contaminação do SAG em território brasileiro (DANTAS et al., 2009; COUTINHO, 2005).

O conceito de qualidade da água é função das condições naturais (escoamento superficial e infiltração no solo), e do uso e da ocupação do solo 
(despejos domésticos ou industriais e aplicação de agrotóxicos no solo) em bacias hidrográficas. Os componentes que alteram o grau de pureza da água são retratados pelas suas características físicas, químicas e biológicas, que são traduzidas como parâmetros de qualidade da água (SPERLING, 2005).

Ainda, pela sua própria natureza, as concentrações de substâncias presentes nas águas subterrâneas são extremamente dependentes do tipo de rocha, solo e/ou estruturas a que estão subordinadas e do tempo de residência da água no aquífero. Desse modo, em determinadas regiões, águas subterrâneas com o teor de um determinado metal acima do considerado normal, para outras regiões, podem estar perfeitamente dentro de uma composição considerada perfeitamente aceitável para aquela determinada região. De maneira geral, devido à ação filtradora lenta, através das camadas permeáveis, velocidade, da ordem de $\mathrm{cm}$ dia-1, as águas subterrâneas deveriam apontar baixos teores de cor, turbidez e isenção de bactérias encontradas em águas superficiais, a não ser que sejam atingidas por alguma fonte poluidora (FREITAS, 1997).

Essa realidade não é diferente no Vale do Rio Pardo, na região central do Estado do Rio Grande do Sul, onde, apesar da ausência de estudos sistemáticos referentes à contaminação da água subterrânea por agrotóxicos e fertilizantes, as zonas de recarga situam-se junto às nascentes dos rios Pardo e Pardinho, regiões de intensa atividade agrícola destacando-se as culturas de fumo e milho.

Nesse contexto, este estudo teve por objetivo avaliar a qualidade das águas subterrâneas nesta bacia, através de variáveis físicas, químicas e microbiológicas, permitindo assim discutir a questão da vulnerabilidade e proteção dos recursos hídricos subterrâneos.

\section{MATERIAL E MÉTODOS}

\section{1 Área de Estudo/Amostragem}

As unidades litológicas aflorantes na bacia hidrográfica do rio Pardo pertencem à parte da sucessão da Bacia do Paraná. Da base para o topo ocorrem - Grupo Rosário do Sul, representado pelas formações Sanga do Cabral, Santa Maria e Caturrita, e o Grupo São Bento, com as formações Botucatu, Serra Geral Facies Gramado e Serra Geral Facies Caxias do Sul sobreposta por depósitos Neogênicos colúvios-aluviais e aluviais (CPRM, 2008) (Figura 1). 


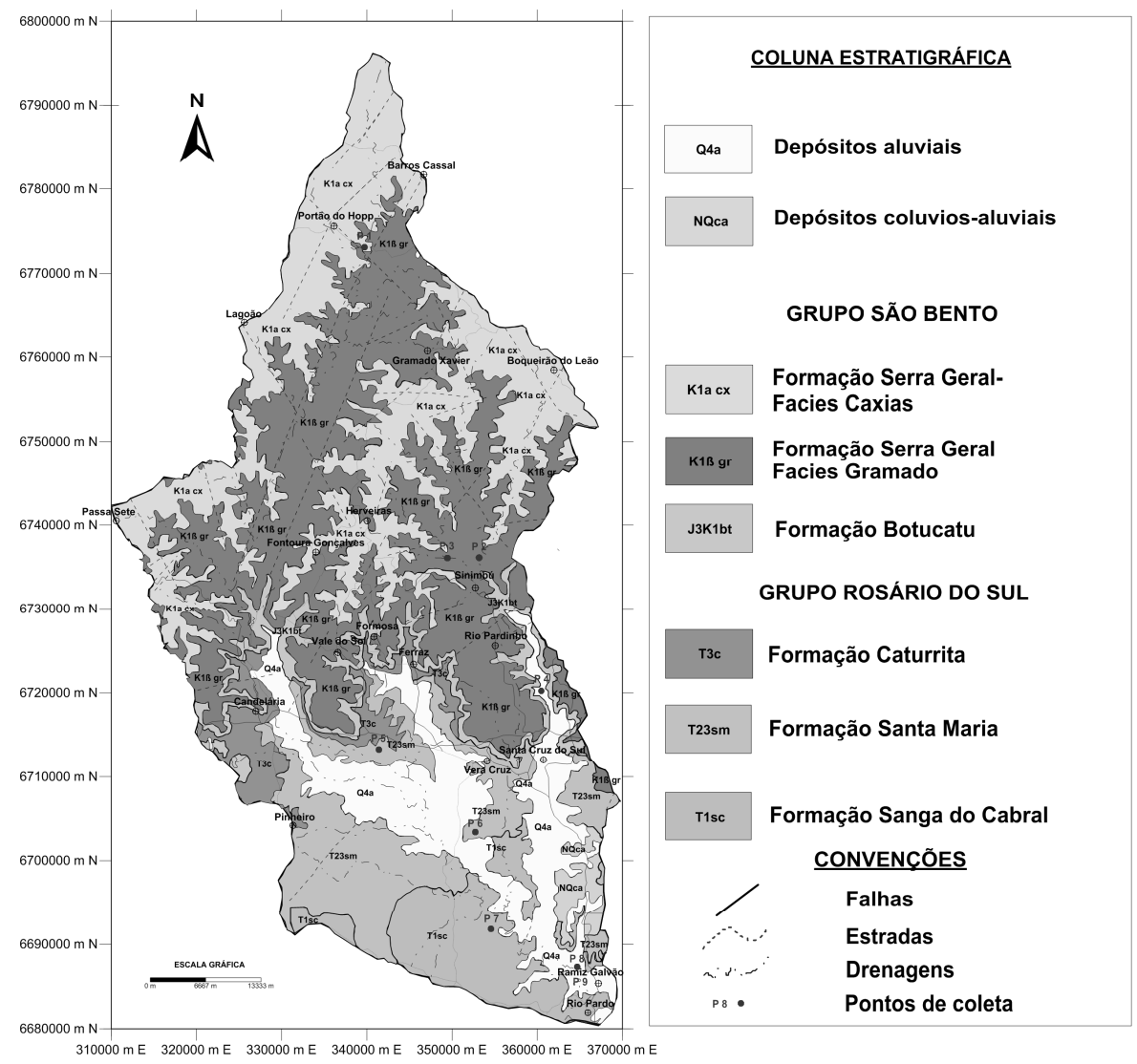

Figura 1. Mapa Geológico bacia hidrográfica do Rio Pardo com pontos de coletas das amostras de água (adaptado de CPRM, 2008).

Ao todo, nove pontos de coleta foram identificados, sendo considerada, dentre os critérios utilizados para localização dos mesmos, a necessidade de que estes locais fossem distribuídos em diferentes contextos geológicos da região, como pode ser observado na Figura 2, possibilitando assim a compreensão do comportamento qualitativo destas águas em cada situação. Foram realizadas cinco campanhas de coleta, que ocorreram nos meses de julho, agosto, setembro, outubro e dezembro de 2009. 


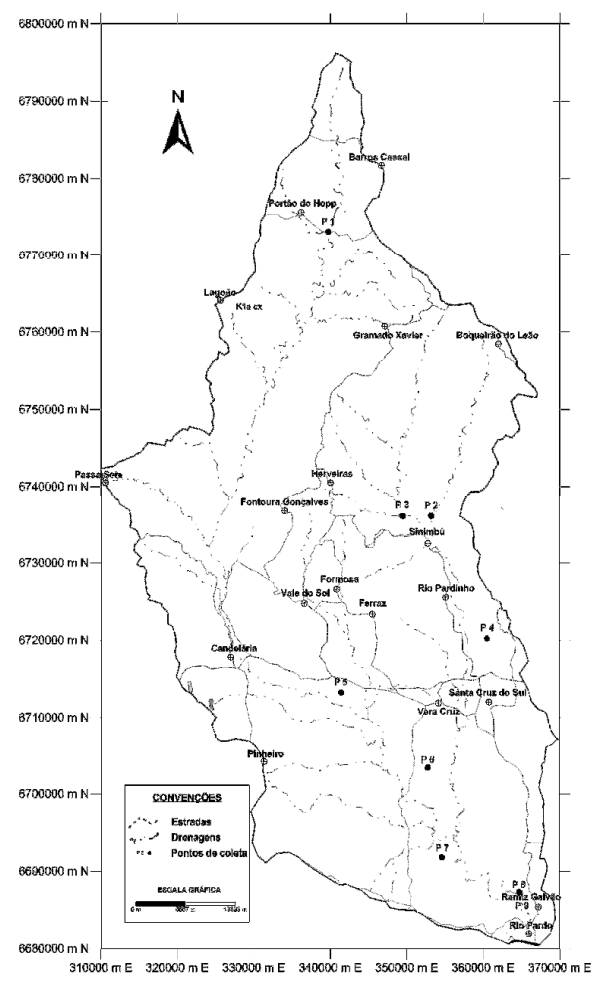

Figura 2. Localização das estações de amostragem de água na Bacia Hidrográfica do Rio Pardo, RS.

As técnicas utilizadas na coleta das amostras, e para as determinações físicas, químicas e microbiológicas, encontram-se descritas no Americam Public Health Association (2005). Na avaliação da qualidade da água utilizou-se a Resolução no 396 do Conselho Nacional do Meio Ambiente (CONAMA, 2008), destacando a Tabela 1 que apresenta os usos indicados em cada classe da referida Resolução.

Tabela 1. Classificação das águas subterrâneas através dos usos (CONAMA, 2008).

Classe Classificação e destino das águas subterrâneas

Especial Preservação de ecossistemas em unidades de conservação de proteção integral e as que contribuam diretamente para os trechos de corpos de água superficial enquadrados como classe especial.

Classe 1 Águas subterrâneas sem alteração de sua qualidade por atividades antrópicas, e que não exigem tratamento para quaisquer usos preponderantes, devido às suas características hidrogeoquímicas naturais.

Classe 2 Águas subterrâneas sem alteração de sua qualidade por atividades antrópicas, e que podem exigir tratamento adequado, dependendo do uso preponderante, devido às suas características hidrogeoquímicas naturais.

Classe 3 Águas subterrâneas com alteração de sua qualidade por atividades antrópicas, para as quais não é necessário o tratamento em função dessas alterações, mas que podem exigir tratamento adequado, dependendo do uso preponderante, devido às suas características hidrogeoquímicas naturais

Classe 4 Águas subterrâneas com alteração de sua qualidade por atividades antrópicas, e que somente possam ser utilizadas, sem tratamento, para o uso preponderante menos restritivo (como recreação de contato secundário).

Classe 5 Águas subterrâneas que possam estar com alteração de sua qualidade por atividades antrópicas, destinadas a atividades que não tenham requisitos de qualidade para uso. 
As variáveis consideradas nesta avaliação bem como os valores limites (VMP - Valor Máximo Permitido) para os usos preponderantes, segundo o Decreto Estadual 12.486, de 20 de outubro de 1978 (SÃO PAULO, 1978), foram alcalinidade de bicarbonatos, alcalinidade de carbonatos e de hidróxidos. Determinaram-se os limites dos parâmetros alumínio, cloro residual livre, coliformes termotolerantes e totais, ferro, manganês, nitrato, nitrogênio amoniacal, $\mathrm{pH}$, sódio, sulfato e sólidos totais dissolvidos (STD), segundo a Portaria no 518, de 25 de março de 2004, do Ministério da Saúde (BRASIL, 2004). O limite considerado para fluoretos teve como base a Portaria $n^{0} 10$, de 16 de agosto de 1999, da Secretaria Estadual da Saúde (RIO GRANDE DO SUL, 1999), considerada a mais restritiva.

\section{RESULTADOS E DISCUSSÕES}

De forma geral, os resultados das determinações físicas, químicas e microbiológicas, nos meses de julho a dezembro de 2009, indicaram que os pontos de coleta apresentam uma alta variabilidade espacial e temporal da estrutura ambiental, destacando-se a Classe 4 de usos da água, que corresponde às águas de má qualidade, basicamente em função das variáveis coliformes totais, coliformes termotolerantes e turbidez, como se pode observar na média dos resultados obtidos, conforme Tabela 2 .

Tabela 2. Média ( \pm desvio-padrão) dos resultados obtidos para cada estação de amostragem de julho a dezembro de 2009.

\begin{tabular}{|c|c|c|c|c|c|c|c|c|c|}
\hline Parâmetros & P1 & P2 & P3 & P4 & P5 & P6 & P7 & P8 & P9 \\
\hline $\begin{array}{c}\text { A.B. } \\
\text { (desvio-padrão) }\end{array}$ & $\begin{array}{c}18,55 \\
( \pm 3,21)\end{array}$ & $\begin{array}{c}50,23 \\
( \pm 2,83)\end{array}$ & $\begin{array}{c}101,48 \\
( \pm 17,73)\end{array}$ & $\begin{array}{c}87,00 \\
( \pm 14,63)\end{array}$ & $\begin{array}{c}119,68 \\
( \pm 34,29)\end{array}$ & $\begin{array}{c}10,63 \\
( \pm 3,67)\end{array}$ & $\begin{array}{c}8,48 \\
( \pm 1,57)\end{array}$ & $\begin{array}{c}117,60 \\
( \pm 0,00)\end{array}$ & $\begin{array}{c}7,23 \\
( \pm 0,25)\end{array}$ \\
\hline $\begin{array}{c}\text { A.C. } \\
\text { (desvio-padrão) }\end{array}$ & $\begin{array}{c}0,00 \\
( \pm 0,00)\end{array}$ & $\begin{array}{c}0,00 \\
( \pm 0,00)\end{array}$ & $\begin{array}{c}0,00 \\
( \pm 0,00)\end{array}$ & $\begin{array}{c}0,00 \\
( \pm 0,00)\end{array}$ & $\begin{array}{c}8,45 \\
( \pm 7,07)\end{array}$ & $\begin{array}{c}0,00 \\
( \pm 0,00)\end{array}$ & $\begin{array}{c}0,00 \\
( \pm 0,00)\end{array}$ & $\begin{array}{c}13,00 \\
( \pm 0,00)\end{array}$ & $\begin{array}{c}0,00 \\
( \pm 0,00)\end{array}$ \\
\hline $\begin{array}{c}\text { A.H. } \\
\text { (desvio-padrão) }\end{array}$ & $\begin{array}{c}0,00 \\
( \pm 0,00)\end{array}$ & $\begin{array}{c}0,00 \\
( \pm 0,00)\end{array}$ & $\begin{array}{c}0,00 \\
( \pm 0,00)\end{array}$ & $\begin{array}{c}0,00 \\
( \pm 0,00)\end{array}$ & $\begin{array}{c}0,00 \\
( \pm 0,00)\end{array}$ & $\begin{array}{c}0,00 \\
( \pm 0,00)\end{array}$ & $\begin{array}{c}0,00 \\
( \pm 0,00)\end{array}$ & $\begin{array}{c}0,00 \\
( \pm 0,00)\end{array}$ & $\begin{array}{c}0,00 \\
( \pm 0,00)\end{array}$ \\
\hline $\begin{array}{c}\text { Alumínio } \\
\text { (desvio-padrão) }\end{array}$ & $\begin{array}{c}1,02 \\
( \pm 0,39) \\
4,28\end{array}$ & $\begin{array}{c}2,90 \\
( \pm 2,07) \\
4,30\end{array}$ & $\begin{array}{c}0,18 \\
( \pm 0,11) \\
9,95\end{array}$ & $\begin{array}{c}9,93 \\
( \pm 5,72) \\
4,73\end{array}$ & $\begin{array}{c}0,11 \\
( \pm 0,09) \\
44,50\end{array}$ & $\begin{array}{c}0,21 \\
( \pm 0,16) \\
3,05\end{array}$ & $\begin{array}{c}0,10 \\
( \pm 0,10) \\
2,95\end{array}$ & $\begin{array}{c}0,10 \\
( \pm 0,00) \\
30,70\end{array}$ & $\begin{array}{c}0,20 \\
( \pm 0,15) \\
3,87\end{array}$ \\
\hline $\begin{array}{l}\text { (desvio-padrão) } \\
\text { (dio }\end{array}$ & $\begin{array}{c}4,28 \\
( \pm 0,94)\end{array}$ & $\begin{array}{c}4,30 \\
( \pm 1,19)\end{array}$ & $( \pm 6,69)$ & $( \pm 0,57)$ & $( \pm 25,06)$ & $( \pm 0,48)$ & $( \pm 1,20)$ & $( \pm 0,00)$ & $( \pm 0,30)$ \\
\hline $\begin{array}{c}\text { C. Termo. } \\
\text { (desvio-padrão) }\end{array}$ & $\begin{array}{c}8,00 \\
( \pm 0,00)\end{array}$ & $\begin{array}{c}8,00 \\
( \pm 0,00)\end{array}$ & $\begin{array}{c}6,20 \\
( \pm 2,70)\end{array}$ & $\begin{array}{c}8,00 \\
( \pm 0,00)\end{array}$ & $\begin{array}{c}8,00 \\
( \pm 0,00)\end{array}$ & $\begin{array}{c}1,85 \\
( \pm 1,31)\end{array}$ & $\begin{array}{c}0,00 \\
( \pm 0,00)\end{array}$ & $\begin{array}{c}0,00 \\
( \pm 0,00)\end{array}$ & $\begin{array}{c}1,10 \\
( \pm 0,78)\end{array}$ \\
\hline $\begin{array}{l}\text { C. Totais } \\
\text { (desvio-padrão) }\end{array}$ & $\begin{array}{c}8,00 \\
( \pm 0,00)\end{array}$ & $\begin{array}{c}8,00 \\
( \pm 0,00)\end{array}$ & $\begin{array}{c}6,87 \\
( \pm 1,70)\end{array}$ & $\begin{array}{c}8,00 \\
( \pm 0,00)\end{array}$ & $\begin{array}{c}4,55 \\
( \pm 3,98)\end{array}$ & $\begin{array}{c}8,00 \\
( \pm 5,66)\end{array}$ & $\begin{array}{c}0,00 \\
( \pm 0,00)\end{array}$ & $\begin{array}{c}0,00 \\
( \pm 0,00)\end{array}$ & $\begin{array}{c}5,30 \\
( \pm 4,08)\end{array}$ \\
\hline $\begin{array}{c}\text { Cl res. Livre } \\
\text { (desvio-padrão) }\end{array}$ & $\begin{array}{c}0,06 \\
( \pm 0,01)\end{array}$ & $\begin{array}{c}0,07 \\
( \pm 0,02)\end{array}$ & $\begin{array}{c}0,05 \\
( \pm 0,00)\end{array}$ & $\begin{array}{c}0,05 \\
( \pm 0,00)\end{array}$ & $\begin{array}{c}0,05 \\
( \pm 0,00)\end{array}$ & $\begin{array}{c}0,05 \\
( \pm 0,00)\end{array}$ & $\begin{array}{c}0,05 \\
( \pm 0,00)\end{array}$ & $\begin{array}{c}0,05 \\
( \pm 0,00)\end{array}$ & $\begin{array}{c}0,05 \\
( \pm 0,00)\end{array}$ \\
\hline $\begin{array}{c}\text { C. E. } \\
\text { (desvio-padrão) }\end{array}$ & $\begin{array}{c}92,95 \\
( \pm 7,56)\end{array}$ & $\begin{array}{l}117,45 \\
( \pm 5,37)\end{array}$ & $\begin{array}{c}246,45 \\
( \pm 57,35)\end{array}$ & $\begin{array}{c}252,25 \\
( \pm 37,68)\end{array}$ & $\begin{array}{c}317,28 \\
( \pm 79,95)\end{array}$ & $\begin{array}{c}99,05 \\
( \pm 37,74)\end{array}$ & $\begin{array}{c}105,30 \\
( \pm 4,05)\end{array}$ & $\begin{array}{c}3500 \\
( \pm 0,00)\end{array}$ & $\begin{array}{c}132,47 \\
( \pm 18,88)\end{array}$ \\
\hline $\begin{array}{c}\text { Ferro } \\
\text { (desvio-padrão) }\end{array}$ & $\begin{array}{c}0,59 \\
( \pm 0,21)\end{array}$ & $\begin{array}{c}1,36 \\
( \pm 0,67)\end{array}$ & $\begin{array}{c}0,13 \\
( \pm 0,12)\end{array}$ & $\begin{array}{c}7,10 \\
( \pm 2,65)\end{array}$ & $\begin{array}{c}0,05 \\
( \pm 0,03)\end{array}$ & $\begin{array}{c}0,09 \\
( \pm 0,09)\end{array}$ & $\begin{array}{c}0,02 \\
( \pm 0,00)\end{array}$ & $\begin{array}{c}0,05 \\
( \pm 0,00)\end{array}$ & $\begin{array}{c}0,08 \\
( \pm 0,03)\end{array}$ \\
\hline $\begin{array}{c}\text { Fluoreto } \\
\text { (desvio-padrão) }\end{array}$ & $\begin{array}{c}0,28 \\
( \pm 0,32)\end{array}$ & $\begin{array}{c}0,08 \\
( \pm 0,01)\end{array}$ & $\begin{array}{c}0,32 \\
( \pm 0,13)\end{array}$ & $\begin{array}{c}0,23 \\
( \pm 0,16)\end{array}$ & $\begin{array}{c}0,25 \\
( \pm 0,27)\end{array}$ & $\begin{array}{c}0,13 \\
( \pm 0,16)\end{array}$ & $\begin{array}{c}0,14 \\
( \pm 0,15)\end{array}$ & $\begin{array}{c}1,60 \\
( \pm 0,00)\end{array}$ & $\begin{array}{c}0,09 \\
( \pm 0,01)\end{array}$ \\
\hline $\begin{array}{c}\text { Magnésio } \\
\text { (desvio-padrão) }\end{array}$ & $\begin{array}{c}2,48 \\
( \pm 0,44)\end{array}$ & $\begin{array}{c}4,33 \\
( \pm 0,54)\end{array}$ & $\begin{array}{c}6,33 \\
( \pm 1,43)\end{array}$ & $\begin{array}{c}9,33 \\
( \pm 1,36)\end{array}$ & $\begin{array}{c}3,50 \\
( \pm 0,64)\end{array}$ & $\begin{array}{c}1,80 \\
( \pm 0,48)\end{array}$ & $\begin{array}{c}1,65 \\
( \pm 0,39)\end{array}$ & $\begin{array}{c}10,00 \\
( \pm 0,00)\end{array}$ & $\begin{array}{c}3,77 \\
( \pm 0,66)\end{array}$ \\
\hline $\begin{array}{c}\text { Manganês } \\
\text { (desvio-padrão) }\end{array}$ & $\begin{array}{c}0,02 \\
( \pm 0,00)\end{array}$ & $\begin{array}{c}0,02 \\
( \pm 0,00)\end{array}$ & $\begin{array}{c}0,02 \\
( \pm 0,00)\end{array}$ & $\begin{array}{c}0,04 \\
( \pm 0,02)\end{array}$ & $\begin{array}{c}0,05 \\
( \pm 0,02)\end{array}$ & $\begin{array}{c}0,04 \\
( \pm 0,01)\end{array}$ & $\begin{array}{c}0,03 \\
( \pm 0,01)\end{array}$ & $\begin{array}{c}0,04 \\
( \pm 0,00)\end{array}$ & $\begin{array}{c}0,08 \\
( \pm 0,07)\end{array}$ \\
\hline $\begin{array}{c}\text { Mat. Org. } \\
\text { (desvio-padrão) }\end{array}$ & $\begin{array}{c}2,00 \\
( \pm 0,00)\end{array}$ & $\begin{array}{c}2,07 \\
( \pm 0,10)\end{array}$ & $\begin{array}{c}2,00 \\
( \pm 0,00)\end{array}$ & $\begin{array}{c}9,73 \\
( \pm 1,41)\end{array}$ & $\begin{array}{c}4,00 \\
( \pm 1,41)\end{array}$ & $\begin{array}{c}3,80 \\
( \pm 2,70)\end{array}$ & $\begin{array}{c}2,00 \\
( \pm 0,00)\end{array}$ & $\begin{array}{c}2,0 \\
( \pm 0,00)\end{array}$ & $\begin{array}{c}2,00 \\
( \pm 0,00)\end{array}$ \\
\hline $\begin{array}{c}\text { Nitrato } \\
\text { (desvio-padrão) }\end{array}$ & $\begin{array}{c}8,40 \\
( \pm 6,48)\end{array}$ & $\begin{array}{c}3,23 \\
( \pm 2,70)\end{array}$ & $\begin{array}{c}5,08 \\
( \pm 4,49)\end{array}$ & $\begin{array}{c}8,60 \\
( \pm 6,66)\end{array}$ & $\begin{array}{c}7,70 \\
( \pm 6,62)\end{array}$ & $\begin{array}{c}8,40 \\
( \pm 6,68)\end{array}$ & $\begin{array}{c}8,78 \\
( \pm 6,57)\end{array}$ & $\begin{array}{c}0,60 \\
( \pm 0,00)\end{array}$ & $\begin{array}{c}6,9 \\
( \pm 6,08)\end{array}$ \\
\hline
\end{tabular}




\begin{tabular}{cccccccccc}
\hline Parâmetros & P1 & P2 & P3 & P4 & P5 & P6 & P7 & P8 & P9 \\
\hline N.amoniacal & 0,50 & 0,50 & 0,50 & 0,50 & 0,50 & 0,57 & 0,50 & 0,5 & 0,50 \\
(desvio-padrão) & $( \pm 0,00)$ & $( \pm 0,00)$ & $( \pm 0,00)$ & $( \pm 0,00)$ & $( \pm 0,00)$ & $( \pm 0,10)$ & $( \pm 0,00)$ & $( \pm 0,00)$ & $( \pm 0,00)$ \\
Ph & 6,37 & 7,46 & 7,46 & 7,30 & 7,98 & 5,25 & 6,10 & 7,72 & 6,07 \\
(desvio-padrão) & $( \pm 0,90)$ & $( \pm 0,32)$ & $( \pm 0,55)$ & $( \pm 0,26)$ & $( \pm 0,16)$ & $( \pm 1,06)$ & $( \pm 0,46)$ & $( \pm 0,00)$ & $( \pm 0,62)$ \\
Potássio & 1,00 & 0,50 & 10,63 & 11,48 & 1,95 & 0,43 & 3,33 & 3,00 & 5,07 \\
(desvio-padrão) & $( \pm 0,12)$ & $( \pm 0,04)$ & $( \pm 18,47)$ & $( \pm 1,67)$ & $( \pm 0,18)$ & $( \pm 0,21)$ & $( \pm 0,20)$ & $( \pm 0,00)$ & $( \pm 0,55)$ \\
Salinidade & 48,23 & 64,03 & 131,43 & 140,20 & 165,70 & 42,80 & 57,23 & 436,00 & 97,57 \\
(desvio-padrão) & $( \pm 25,43$ & $( \pm 36,41$ & $( \pm 69,32)$ & $( \pm 79,42)$ & $( \pm 91,72)$ & $( \pm 21,90)$ & $( \pm 31,42$ & $( \pm 0,00)$ & $( \pm 31,48)$ \\
& & ( & & & & & ) & & \\
STD & 67,4 & 85,16 & 178,71 & 182,88 & 230,03 & 71,71 & 76,35 & 853,10 & 95,89 \\
(desvio-padrão) & $( \pm 5,49)$ & $( \pm 3,89)$ & $( \pm 41,56)$ & $( \pm 27,32)$ & $( \pm 57,97)$ & $( \pm 27,41)$ & $( \pm 2,95)$ & $( \pm 0,00)$ & $( \pm 13,43)$ \\
Sódio & 4,15 & 4,58 & 11,66 & 45,38 & 12,18 & 7,33 & 8,25 & 951,80 & 7,20 \\
(desvio-padrão) & $( \pm 0,34)$ & $( \pm 0,33)$ & $( \pm 9,58)$ & $( \pm 62,96)$ & $( \pm 2,19)$ & $( \pm 1,29)$ & $( \pm 0,56)$ & $( \pm 0,00)$ & $( \pm 0,84)$ \\
Sulfato & 5,56 & 10,88 & 10,15 & 3,60 & 13,48 & 4,25 & 1,00 & 2537,50 & 2,40 \\
(desvio-padrão) & $( \pm 2,11)$ & $( \pm 4,05)$ & $( \pm 9,77)$ & $( \pm 2,65)$ & $( \pm 4,36)$ & $( \pm 1,76)$ & $( \pm 0,00)$ & $( \pm 0,00)$ & $( \pm 1,97)$ \\
Turbidez & 15,23 & 28,73 & 3,89 & 100,4 & 3,40 & 7,95 & 5,37 & 2,78 & 5,71 \\
(desvio-padrão) & $( \pm 1,49)$ & $( \pm 11,78$ & $( \pm 3,40)$ & $( \pm 25,81)$ & $( \pm 2,63)$ & $( \pm 3,64)$ & $( \pm 3,66)$ & $( \pm 0,00)$ & $( \pm 4,38)$ \\
& & & & & & & & & \\
\hline Classe & $\mathbf{4}$ & $\mathbf{4}$ & $\mathbf{4}$ & $\mathbf{4}$ & $\mathbf{4}$ & $\mathbf{4}$ & $\mathbf{2}$ & $\mathbf{4}$ & $\mathbf{4}$ \\
\hline
\end{tabular}

Onde: A.B. - Alcalinidade bicarbonatos; A.C. - Alcalinidade carbonatos; A.H. Alcalinidade hidróxidos; $\mathrm{Cl}$ res. livre - Cloro residual livre; C.Termo. - Coliformes termotolerantes (NMP $100 \mathrm{~mL}-1$ ); C. Totais - Coliformes totais (NMP $100 \mathrm{~mL}-1$ ); C. E. Condutividade elétrica; Mat. Org. - Matéria orgânica; N. amoniacal - Nitrogênio amoniacal; STD - Sólidos totais dissolvidos. Unidades em $\mathrm{mg} \mathrm{L-1}$, exceto para C.Termotolerantes e C. Totais.

Quanto aos resultados para coliformes termotolerantes, as estações de amostragem de poços rasos ( $<6 \mathrm{~m})(\mathrm{P} 1, \mathrm{P} 2, \mathrm{P} 4, \mathrm{P} 5$ e P6), enquadraram-se na Classe 4 (Figura 3), destacando que a resolução 396/2008 do CONAMA define que a ausência de coliformes é o limite para diferenciar a Classe 4 das outras. Da mesma forma, as estações de amostragem P3 e P9, de poços profundos (> $6 \mathrm{~m}$ ), enquadraram-se na Classe 4.

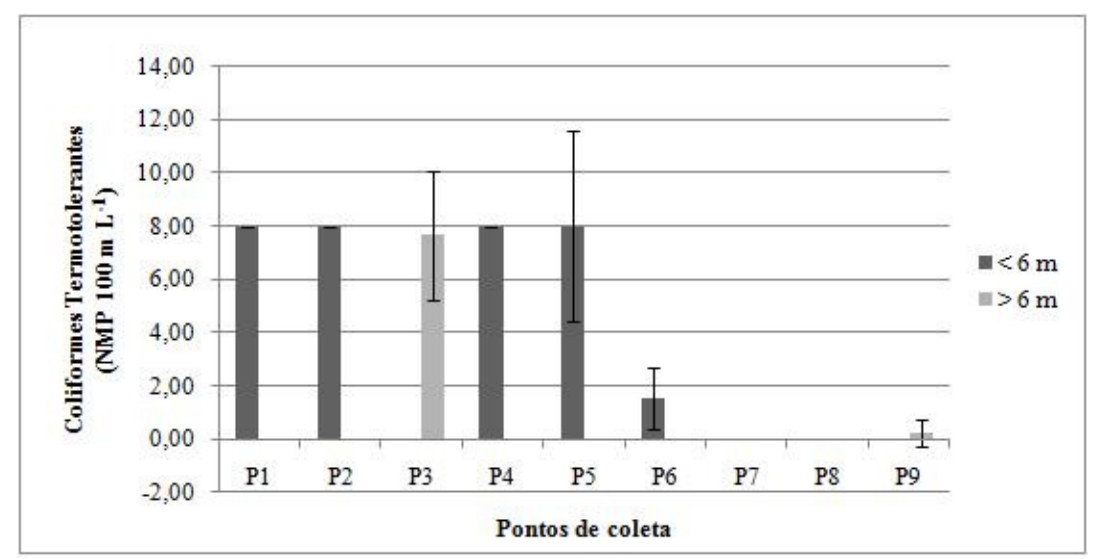

Figura 3. Avaliação da qualidade da água utilizando os valores médios ( \pm desviopadrão) de coliformes termotolerantes, nos pontos de coleta em poços $>6 \mathrm{~m}$ e $<6 \mathrm{~m}$, distribuídos ao longo da bacia hidrográfica do rio Pardo, RS, Brasil.

Devido às atividades antrópicas comprometerem significativamente os aquíferos da região, apresenta-se a seguir as fontes potenciais de contaminação identificadas durante o desenvolvimento deste estudo: 
a) Perfuração de poços sem a elaboração de projetos construtivos, anotação de responsabilidade técnica e registro do poço junto aos órgãos competentes. A inadequada construção, sem vedação sanitária, de poços rasos (P1, P2, P4, P5 e P6) pode torná-los fontes pontuais de contaminação das águas subterrâneas pela conexão direta que eles proporcionam entre a superfície e as porções mais rasas do aquífero com as partes mais profundas.

b) $\mathrm{O}$ uso de insumos agrícolas, como agrotóxicos e fertilizantes, tem grande potencial de contaminação difusa. Valores elevados desta variável foram observados nos meses de julho e agosto (P1, P4, P5, P6, P7 e P9), meses em que houve menor volume de chuvas.

Na sua forma natural, as principais restrições ao consumo, identificadas neste estudo, foram:

a) Problemas localizados de elevada dureza e/ou sólidos totais dissolvidos nas regiões de ocorrência de lentes de rochas calcárias, como, por exemplo, no ponto P5 localizado na Formação Santa Maria.

b) Elevados valores de sólidos totais dissolvidos nas porções mais profundas dos aquíferos, especialmente nas partes confinadas das bacias sedimentares, como é o caso do P8, localizado em sistema de depósitos aluviais.

c) Ocorrência natural nas rochas de minerais cuja dissolução, localmente, gera águas com concentrações acima do padrão de potabilidade. É o caso do ferro e do alumínio nos pontos P1, P2 e P4. Este fato também pode ocasionar um aumento na turbidez, visto que no caso do excesso de ferro pode produzir uma cor amarelada à água. Diversos estudos realizados no Estado do Rio Grande do Sul (LOBO et al., 2000; COSTA et al., 2004) têm mostrado elevados valores de íons fluoreto nas águas subterrâneas. Nesta pesquisa observou-se excesso desse mineral apenas no ponto denominado P8 (ponto amostrado apenas no mês de julho de 2009).

\section{CONSIDERAÇÕES FINAIS}

Os resultados deste estudo indicaram que a qualidade das águas dos poços com profundidade inferior a 6 metros apresentam-se, de fato, mais vulneráveis devido a alterações antrópicas, condição evidenciada a partir das variáveis coliformes totais, coliformes termotolerantes, nitrato e turbidez, cujas concentrações impossibilitam a utilização destas águas para usos preponderantes. As elevadas concentrações de turbidez ocorreram basicamente pelo excesso de chuvas ocorrido no período de setembro a dezembro de 2009, fator também significativo na alteração da variável nitrato, diminuindo sua concentração neste período por efeito da diluição. Entretanto, as elevadas concentrações de ferro e alumínio seriam características naturais de dissolução de rochas. 
Já a qualidade das águas de poços profundos depende basicamente de suas características hidrogeológicas e hidrogeoquímicas naturais, em função das variáveis salinidade, sódio e sulfato. Contudo, estas águas também apresentaram contaminação por atividades antrópicas, basicamente em função das elevadas concentrações de nitrato, nos meses de julho e agosto de 2009 nos pontos P1, P4, P5, P6, P7 e P9, e coliformes termotolerantes, P1, P2, P3, P4, P5, P6 e P9.

\section{REFERÊNCIAS}

AMERICAN PUBLIC HEALTH ASSOCIATION. APHA. Standard Methods for the Examination of water and Wastewater. 21 ed. Washington. 2005.

BORGHETTI, N. B.; BORGGHETTI, J. R.; ROSA FILHO, E. F. Aquífero Guarani: A Verdadeira Integração dos Países do Mercosul. Curitiba: Ed. Roberto Marinho, 2004.

BRASIL. Portaria no 518, de 25 de março de 2004 do Ministério da Saúde. Diário Oficial, n. 59, de 26 de março de 2004.

CONAMA. Conselho Nacional do Meio Ambiente. Resolução n 396, de 3 de abril de 2008. Publicada no Diário Oficial da União nº 66, de 7 de abril de 2008.

COSTA, A. B.; LOBO, E. A.; KIRST, A.; SOARES, J.; GOETTEMS, C. H. Estudo comparativo da concentração de flúor, $\mathrm{pH}$ e condutividade elétrica da água subterrânea dos municípios de Santa Cruz do Sul, Venâncio Aires e Vera Cruz, RS, Brasil. In: XIII CONGRESSO BRASILEIRO DE ÁGUAS SUBTERRÂNEAS, 2004, Cuiabá. Anais..., Cuiabá: ABAS, 2004, CD-ROM.

COUTINHO, C. F. B., TANIMOTO, S. T., GALLI, A., GARBELLINI, G. S., TAKAYAMA, M., AMARAL R. B., MAZO, L. H., AVACA, L. A., MACHADO, S. A. S. Pesticidas: Mecanismo de ação, degradação e toxidez. Revista Ecotoxicologia e Meio Ambiente, Curitiba, 5: 65-72. 2005.

CPRM. Serviço Geológico do Brasil. Mapa Geológico do Rio Grande do Sul. Escala: 1:750.000. 2008, CD-ROM.

DANTAS, A. B.; PASCHOALATO, C. P. R.; BALLEJO, R. R.;DI BERNARDO, L. Préoxidação e adsorção em carvão ativado granular para remoção dos herbicidas Diuron e Hexazinona de água subterránea. Eng. Sanit. Ambient. [online]. v.14, n. 3, p. 373- 380. 2009.

FREITAS, A. L. S. Água Subterrânea na Legislação Brasileira de Recursos Hídricos. Associação Brasileira de Educação Agrícola Superior. 75p. 1997.

LOBO, E. A.; BEN DA COSTA, A.; KIRST, A. Qualidade das águas subterrâneas na Região do Vale do Rio Pardo e Rio Taquari, RS, Brasil. In: CONGRESSO MUNDIAL INTEGRADO DE ÁGUAS SUBTERRÂNEAS, 1. 2000, Fortaleza. Anais..., Fortaleza: ABAS. CD-ROM. 2000. 
RIO GRANDE DO SUL. Portaria no 10, de 16 de agosto de 1999, da Secretaria Estadual da Saúde, do Estado do Rio Grande do Sul. Diário Oficial do Estado do Rio Grande do Sul, de 27 de agosto de 1999.

SÃO PAULO. Decreto Estadual 12.486, de 20 de outubro de 1978. Norma Técnica Alimentar N. 60 (NTA-60). 1978.

SPERLING, M. V. Introdução à qualidade das águas e ao tratamento de esgotos. Belo Horizonte: Editora,2005, p. 443. 